\title{
Long time behavior of the two-dimensional Vlasov equation with a strong external magnetic field
}

\author{
Emmanuel Frénod and Eric Sonnendrücker
}

$\mathbf{N}^{\circ} 3428$

Mai 1998

THÈME 4

\section{apport \\ de recherche}





\title{
Long time behavior of the two-dimensional Vlasov equation with a strong external magnetic field
}

\author{
Emmanuel Frénod* and Eric Sonnendrücker ${ }^{\dagger}$ \\ Thème 4 - Simulation et optimisation \\ de systèmes complexes \\ Projet Numath
}

Rapport de recherche $\mathrm{n}^{\circ} 3428$ - Mai 1998 - 15 pages

\begin{abstract}
When charged particles are submitted to a large external magnetic field, their movement in first approximation occurs along the magnetic field lines and obeys a one dimensional Vlasov equation along these field lines. However, when observing the particles on a sufficiently long time time scale, a drift phenomenon perpendicular to the magnetic field lines superposes to this first movement. In this report, we present a rigorous asymptotic analysis of the two-dimensional Vlasov equation when the magnetic field tends to infinity, the observation time scale growing accordingly. Techniques based on the twoscale convergence and the introduction of a second problem enable us to find an equation verified by the weak limit of the distribution function.
\end{abstract}

Key-words: Vlasov-Poisson equations, kinetic equations, homogenization, gyrokinetic approximation, multiple time scales, two scale convergence.

* LMAM, Université de Bretagne Sud, Centre Universitaire de Vannes, 1 rue de la loi, F-56000 Vannes.

† Institut Elie Cartan, Université Henri Poincaré - Nancy 1, F-54506 Vandœuvre-lès-Nancy Cedex. Unité de recherche INRIA Lorraine

Technopôle de Nancy-Brabois, Campus scientifique, 615 rue de Jardin Botanique, BP 101, 54600 VILLERS LÈS NANCY (France)

Téléphone : (33) 0383593030 - Télécopie : (33) 0383278319

Antenne de Metz, technopôle de Metz 2000, 4 rue Marconi, 55070 METZ

Téléphone : (33) 0387203500 - Télécopie : (33) 0387763977 


\section{Comportement en temps long de l'équation de Vlasov bidimensionnelle avec un fort champ magnétique extérieur}

Résumé : Lorsque des particules chargées sont soumises à un fort champ magnétique extérieur, leur déplacement en première approximation a lieu le long des lignes de champ magnétique et est régi par une une équation de Vlasov unidimensionnelle le long de ces lignes de champ. Néanmoins, en observant les particules sur un temps suffisamment long, il vient ce rajouter à ce phénomène un effet de dérive perpendiculaire à ces lignes de champ magnétique. Nous présentons dans ce rapport, une étude asymptotique rigoureuse de l'équation de Vlasov bidimensionnelle lorsque le champ magnétique extérieur tend vers l'infini, le temps d'observation s'allongeant en même temps. Des techniques basées sur la méthode de convergence à deux échelles et l'introduction d'un problème annexe, nous permettent de trouver une équation vérifiée par la limite faible de la fonction de distribution.

Mots-clé : $\quad$ Equations de Vlasov-Poisson, équations cinétiques, homogénéisation, approximation gyrocinétique, échelles de temps multiples, convergence à deux échelles. 


\section{Introduction}

Following our first paper [5], we continue the investigation of the Vlasov equation with a strong external magnetic field. This problem is of great importance in many plasma devices like electron guns, diodes and most of all tokamaks. In the first paper, we proved that in the limit when the magnetic field goes to infinity, the three-dimensional Vlasov equation reduces to a one dimensional equation along the magnetic field lines. In a device like the tokamak the geometry of which is toroidal, the particles then circle around the device. But when watching the particles on a long enough time scale, if those are submitted to a self consistent or external electric field, a drift phenomenon perpendicular to the magnetic field occurs and this is what we want to investigate in this paper.

In order to describe this behavior, we consider a strong magnetic field $\frac{\mathcal{M}}{\varepsilon}$, where $\varepsilon$ is a small parameter and with $\mathcal{M}=\mathbf{e}_{1}$ where $\mathbf{e}_{1}$ is the first vector of the canonical basis of $I R^{3}\left(\mathbf{e}_{1}, \mathbf{e}_{2}, \mathbf{e}_{3}\right)$. We also assume that the charged particles are submitted to an external electric field deriving from a potential the derivative of which vanishes in the direction of the magnetic field. This assumption which is somewhat restrictive enables us to decouple the two transverse directions from the parallel direction and thus consider only what happens in the plane orthogonal to the magnetic field, which is a simplified description of the physical problem. Moreover, the observation time scale we consider is $\frac{1}{\varepsilon}$.

In the context we chose, no force acts on the particles in the $x_{1}$-direction. Hence the problem may be modeled by a two-dimensional Vlasov-equation. The natural position variable is then $\mathbf{x}=\left(x_{2}, x_{3}\right) \in \mathbb{R}_{x}^{2}$, the velocity variable is $\mathbf{v}=\left(v_{2}, v_{3}\right) \in \mathbb{R}_{v}^{2}$ and the time space is $[0, T)$. We introduce the notation $\Omega=\mathbb{R}_{x}^{2} \times \mathbb{R _ { v } ^ { 2 }}, \mathcal{O}=[0, T) \times \mathbb{R}_{x}^{2}$ and $\mathcal{O}=[0, T) \times \Omega$.

Now, the space-velocity distribution $f_{s}^{\varepsilon}(t, \mathbf{x}, \mathbf{v})$, describing the above phenomenon for a particle species $s$ is the solution of:

$$
\left\{\begin{array}{l}
\frac{\partial f_{s}^{\varepsilon}}{\partial t}+\frac{\mathbf{v}}{\varepsilon} \cdot \nabla_{x} f_{s}^{\varepsilon}+\lambda_{s}\left(\frac{-\nabla_{x} p}{\varepsilon}+\mathbf{v} \times \frac{\mathcal{M}}{\varepsilon^{2}}\right) \cdot \nabla_{v} f_{s}^{\varepsilon}=0 \\
f_{s \mid t=0}^{\varepsilon}=f_{s 0}
\end{array}\right.
$$

where $\lambda_{s}$ is a dimensionless constant linked to the charge over mass ratio of the species $s$ and thus characterizing this species of particles and where

$$
\mathbf{v} \times \mathcal{M}=\left(\begin{array}{c}
v_{3} \\
-v_{2}
\end{array}\right),
$$

$p(t, \mathbf{x}) \in C^{1}\left(\mathbb{R}_{t}, C_{b}^{2}\left(\mathbb{R}_{x}^{2}\right)\right), C_{b}^{2}$ being the space of bounded and twice continuously differentiable functions. The initial data satisfies

$$
f_{s_{0}} \geq 0, \quad \int_{\Omega} f_{s_{0}} d \mathbf{x} d \mathbf{v}<\infty, \quad \int_{\Omega} f_{s_{0}}^{2} d \mathbf{x} d \mathbf{v}<\infty .
$$

With those conditions, equation (1.1) has for any positive $\varepsilon$ a solution which is positive, which belongs to $L^{\infty}\left(0, T ; L^{1} \cap L^{2}(\Omega)\right)$ and which satisfies

$\operatorname{RR} \mathrm{n}^{\circ} 3428$

$$
\left\|f_{s}^{\varepsilon}\right\|_{L^{\infty}\left(0, T ; L^{2}(\Omega)\right)} \leq C,
$$


for some constant $C$.

From (1.3), we may deduce that we can extract a subsequence such that

$$
f_{s}^{\varepsilon} \rightarrow f_{s} \text { in } L^{\infty}\left(0, T ; L^{2}(\Omega)\right) \text { weak }-*
$$

but also, see $[1,9]$, for any $\psi(t, \tau, \mathbf{x}, \mathbf{v})$, regular, with compact support in $t, \mathbf{x}, \mathbf{v}$ and $2 \pi / \lambda_{s}$-periodic in $\tau$, we have that there exists a profile $F_{s}(t, \tau, \mathbf{x}, \mathbf{v}) \in L^{\infty}\left(0, T ; L_{2 \pi / \lambda_{s}}^{\infty}\left(\mathbb{R}_{\tau}\right.\right.$; $\left.L^{2}(\Omega)\right)$ ), also called the two scale limit of $f_{s}^{\varepsilon}$, such that

$$
\int_{\mathcal{Q}} f_{s}^{\varepsilon}(\psi)^{\varepsilon} d t d \mathbf{x} d \mathbf{v} \rightarrow \int_{\mathcal{Q}} \int_{0}^{2 \pi / \lambda_{s}} F_{s} \psi d \tau d t d \mathbf{x} d \mathbf{v}
$$

where $(\psi)^{\varepsilon}=\psi\left(t, \frac{t}{\varepsilon^{2}}, \mathbf{x}, \mathbf{v}\right)$. Moreover the weak-* limit $f_{s}$ of $\left(f_{s}^{\varepsilon}\right)_{\varepsilon}$ is expressed in term of the two scale limit $F_{s}$

$$
f_{s}=\int_{0}^{2 \pi / \lambda_{s}} F_{s}(., \tau, ., .) d \tau
$$

Notice that we adopt the following convention: if $\lambda_{s}<0$, then $F_{s} \leq 0$.

Using this tool we shall derive the equation verified by $F_{s}$ and then formula (1.6) will give us the equation for $f_{s}$ for each species $s$ of particles. Moreover, those equations will no more be genuine kinetic equations, but merely involve $\mathbf{v}$ as a parameter. Hence we shall be able, integrating over the velocity variable $\mathbf{v}$, to obtain one unique equation satisfied by the charge density

$$
\rho=\sum_{s} q_{s} \int_{\mathbb{R}_{v}^{2}} f_{s} d \mathbf{v}
$$

of the whole bunch of particles, where $q_{s}$ is the dimensionless charge of species $s$.

Let us now state the main results of the paper:

THEOREM 1.1 Under assumptions (1.2), the sequence $\left(f_{s}^{\varepsilon}(t, \mathbf{x}, \mathbf{v})\right)_{\varepsilon}$ of solutions of (1.1) weak-* converges to $f_{s}(t, \mathbf{x}, \mathbf{v})$ unique solution of

$$
\left\{\begin{array}{l}
\frac{\partial f_{s}}{\partial t}+\left(-\nabla_{x} p \times \mathcal{M}\right) \cdot \nabla_{x} f_{s}+\frac{1}{2}(\Delta p(\mathbf{v} \times \mathcal{M})) \cdot \nabla_{v} f_{s}=0 \\
f_{s \mid t=0}=\frac{1}{2 \pi} \int_{0}^{2 \pi} f_{s 0}(\mathbf{x}, \mathbf{r}(\mathbf{v}, \tau)) d \tau
\end{array}\right.
$$

where $\mathbf{r}(\mathbf{v}, \tau)$ is the rotation of angle $\tau$ applied to $\mathbf{v}$.

\section{Moreover}

TheOREM 1.2 Assuming (1.2) satisfied for a set of particles, the charge density $\rho$ defined by (1.7) of the set of particles is the unique solution of

$$
\left\{\begin{array}{l}
\frac{\partial \rho}{\partial t}+\left(-\nabla_{x} p \times \mathcal{M}\right) \cdot \nabla_{x} \rho=0 \\
\rho_{\mid t=0}=\sum_{s} q_{s} \int_{\mathbb{R}_{v}^{2}} f_{s 0}(\mathbf{x}, \mathbf{v}) d \mathbf{v} d \tau
\end{array}\right.
$$


This model corresponds to the guiding-center model which has been used by physicist for some time $[4,6,8,10]$. We are now proceeding with the demonstration of these results, which is outlined in the following way: First we determine a constraint equation and study its consequences, then we derive an equation enabling to compute the two-scale limit of $f_{s}^{\varepsilon}$, and finally we give an equation for the weak limit of $f_{s}^{\varepsilon}$ and prove the theorems.

\section{The constraint equation and its consequences}

We multiply equation (1.1), where we do not write down explicitely for the moment the index $s$, by $(\psi)^{\varepsilon}=\psi\left(t, \frac{t}{\varepsilon^{2}}, \mathbf{x}, \mathbf{v}\right)$ where $\psi(t, \tau, \mathbf{x}, \mathbf{v})$ is regular, with compact support in $(t, \mathbf{x}, \mathbf{v})$ and $2 \pi / \lambda$-periodic in $\tau$. We then integrate by parts and get the following weak formulation with oscillating test functions:

$$
\begin{aligned}
\int_{\mathcal{Q}} f^{\varepsilon}\left[\left(\frac{\partial \psi}{\partial t}\right)^{\varepsilon}+\frac{1}{\varepsilon^{2}}\left(\frac{\partial \psi}{\partial \tau}\right)^{\varepsilon}+\frac{\mathbf{v}}{\varepsilon} \cdot\left(\nabla_{x} \psi\right)^{\varepsilon}+\lambda\left(\frac{-\nabla_{x} p}{\varepsilon}+\right.\right. & \left.\left.\mathbf{v} \times \frac{\mathcal{M}}{\varepsilon^{2}}\right) \cdot\left(\nabla_{v} \psi\right)^{\varepsilon}\right] d t d \mathbf{x} d \mathbf{v} \\
& =-\int_{\Omega} f_{0} \psi(0,0, ., .) d \mathbf{x} d \mathbf{v}
\end{aligned}
$$

Multiplying (2.1) by $\varepsilon^{2}$ and passing to the limit gives

$$
\int_{\mathcal{Q}} \int_{0}^{2 \pi / \lambda} F\left(\frac{\partial \psi}{\partial \tau}+\lambda(\mathbf{v} \times \mathcal{M}) \cdot \nabla_{v} \psi\right) d \tau d t d \mathbf{x} d \mathbf{v}=0
$$

which is as we saw in Frénod and Sonnendrücker [5] equivalent to

$$
\frac{\partial F}{\partial \tau}+\lambda(\mathbf{v} \times \mathcal{M}) \cdot \nabla_{v} F=0 \text { in } \mathcal{D}^{\prime}\left(\mathbb{R}_{\tau} \times \mathbb{R}_{v}^{2}\right),
$$

for almost every $t \in[0, T)$ and almost every $\mathbf{x} \in \mathbb{R}_{x}^{2}$.

We now introduce the transformation $\mathbf{r}(., \tau): I R^{2} \rightarrow \mathbb{R}^{2}$ defined by

$$
\mathbf{r}(\mathbf{v}, \tau)=\left[v_{2} \cos \tau-v_{3} \sin \tau\right] \mathbf{e}_{2}+\left[v_{2} \sin \tau+v_{3} \cos \tau\right] \mathbf{e}_{3} .
$$

From (2.3) we may deduce (see [5]) that there exists a function

$$
G \equiv G(t, \mathbf{x}, \mathbf{u}) \in L^{\infty}\left(0, T ; L^{2}\left(\mathbb{R}_{x}^{2} \times I R_{u}^{2}\right)\right),
$$

such that

$$
F(t, \tau, \mathbf{x}, \mathbf{v})=G(t, \mathbf{x}, \mathbf{r}(\mathbf{v}, \lambda \tau))
$$

where we denote, similarly to $\mathbf{x}$ and $\mathbf{v}, \mathbf{u}=\left(u_{2}, u_{3}\right)$.

\section{Obtention of an equation for $\mathrm{G}$}

In order to deduce the equation satisfied by $G$, we shall use first, in the weak formulation, test functions satisfying the constraint. This will yield a weak formulation in which one $\operatorname{RR} \mathrm{n}^{\circ} 3428$ 
term is multiplied by $\frac{1}{\varepsilon}$. Choosing then appropriate test functions and using an ad hoc change of variables, we shall obtain the limit of this term. The deduction of the equation for $G$ will then be straightforward.

For any function $\varphi(t, \mathbf{x}, \mathbf{u})$ regular and with compact support, we define $\psi(t, \tau, \mathbf{x}, \mathbf{v})=$ $\varphi(t, \mathbf{x}, \mathbf{r}(\mathbf{v}, \lambda \tau))$. Then the function $\psi$ is $2 \pi / \lambda$-periodic, satisfies the constraint, and using $(\psi)^{\varepsilon}$ as a test function in (2.1) leads to:

$$
\int_{\mathcal{Q}} f^{\varepsilon}\left[\left(\frac{\partial \psi}{\partial t}\right)^{\varepsilon}+\frac{\mathbf{v}}{\varepsilon} \cdot\left(\nabla_{x} \psi\right)^{\varepsilon}+\lambda\left(\frac{-\nabla_{x} p}{\varepsilon}\right) \cdot\left(\nabla_{v} \psi\right)^{\varepsilon}\right] d t d \mathbf{x} d \mathbf{v}=-\int_{\Omega} f_{0} \psi(0,0, ., .) d \mathbf{x} d \mathbf{v} .
$$

We now introduce the change of variables

$$
\mathbf{v} \rightarrow \mathbf{u}=\mathbf{r}\left(\mathbf{v}, \lambda \frac{t}{\varepsilon^{2}}\right)
$$

whose reverse transformation is

$$
\mathbf{u} \rightarrow \mathbf{v}=\mathbf{r}\left(\mathbf{u},-\lambda \frac{t}{\varepsilon^{2}}\right) .
$$

Using the definition of $\psi$, and defining $g^{\varepsilon}(t, \mathbf{x}, \mathbf{u})$ by $g^{\varepsilon}(t, \mathbf{x}, \mathbf{u})=f^{\varepsilon}\left(t, \mathbf{x}, \mathbf{r}\left(\mathbf{u},-\lambda \frac{t}{\varepsilon^{2}}\right)\right)$ which is equivalent to $f^{\varepsilon}(t, \mathbf{x}, \mathbf{v})=g^{\varepsilon}\left(t, \mathbf{x}, \mathbf{r}\left(\mathbf{v}, \lambda \frac{t}{\varepsilon^{2}}\right)\right)$, since

$$
\begin{gathered}
\frac{\partial \psi}{\partial v_{2}}(t, \tau, \mathbf{x}, \mathbf{v})=\cos \lambda \tau \frac{\partial \varphi}{\partial u_{2}}(t, \mathbf{x}, \mathbf{r}(\mathbf{v}, \tau))+\sin \lambda \tau \frac{\partial \varphi}{\partial u_{3}}(t, \mathbf{x}, \mathbf{r}(\mathbf{v}, \lambda \tau)) \\
\frac{\partial \psi}{\partial v_{3}}(t, \lambda \tau, \mathbf{x}, \mathbf{v})=-\sin \lambda \tau \frac{\partial \varphi}{\partial u_{2}}(t, \mathbf{x}, \mathbf{r}(\mathbf{v}, \lambda \tau))+\cos \lambda \tau \frac{\partial \varphi}{\partial u_{3}}(t, \mathbf{x}, \mathbf{r}(\mathbf{v}, \lambda \tau))
\end{gathered}
$$

the change of variables (3.2) transforms the equation (3.1) into

$$
\begin{aligned}
& \int_{\mathcal{Q}^{\prime}} g^{\varepsilon}(t, \mathbf{x}, \mathbf{u})\left[\frac{\partial \varphi}{\partial t}(t, \mathbf{x}, \mathbf{u})\right. \\
& +\frac{1}{\varepsilon}\left\{\left(u_{2} \cos \lambda \frac{t}{\varepsilon^{2}}+u_{3} \sin \lambda \frac{t}{\varepsilon^{2}}\right) \frac{\partial \varphi}{\partial x_{2}}(t, \mathbf{x}, \mathbf{u})+\left(-u_{2} \sin \lambda \frac{t}{\varepsilon^{2}}+u_{3} \cos \lambda \frac{t}{\varepsilon^{2}}\right) \frac{\partial \varphi}{\partial x_{3}}(t, \mathbf{x}, \mathbf{u})\right. \\
& +\lambda\left[\left(\left(-\partial_{x_{2}} p\right) \cos \lambda \frac{t}{\varepsilon^{2}}+\left(\partial_{x_{3}} p\right) \sin \lambda \frac{t}{\varepsilon^{2}}\right) \frac{\partial \varphi}{\partial u_{2}}(t, \mathbf{x}, \mathbf{u})\right. \\
& \left.\left.\left.+\left(\left(-\partial_{x_{2}} p\right) \sin \lambda \frac{t}{\varepsilon^{2}}+\left(-\partial_{x_{3}} p\right) \cos \lambda \frac{t}{\varepsilon^{2}}\right) \frac{\partial \varphi}{\partial u_{3}}(t, \mathbf{x}, \mathbf{u})\right]\right\}\right] d t d \mathbf{x} d \mathbf{v} \\
& =-\int_{\Omega} f_{0} \psi(0,0, \mathbf{x}, \mathbf{v}) d \mathbf{x} d \mathbf{v}=-\int_{\Omega} f_{0} \varphi(0, \mathbf{x}, \mathbf{u}) d \mathbf{x} d \mathbf{u}
\end{aligned}
$$

Now we focus, for a few pages, on passing to the limit in (3.4). For this purpose, we need to characterize the weak-* limit and the two scale limit of $g^{\varepsilon}$, and to compute the limit of the term containing the $\frac{1}{\varepsilon}$ in factor.

First, we have

Lemma 3.1 The two scale limit of $g^{\varepsilon}$ is the function $G$ defined by (2.5). Consequently, the weak-* limit $g$ of $g^{\varepsilon}$ is $(2 \pi / \lambda) G$.

INRIA 
Proof. We take any function $\varphi(t, \tau, \mathbf{x}, \mathbf{u})$, regular, with compact support in $(t, \mathbf{x}, \mathbf{u})$ and $2 \pi / \lambda$-periodic in $\tau$. On the one hand, denoting by $\bar{G}$ the two scale limit of $g^{\varepsilon}$, we have

$$
\int_{\mathcal{Q}^{\prime}} g^{\varepsilon}(t, \mathbf{x}, \mathbf{u}) \varphi\left(t, \frac{t}{\varepsilon^{2}}, \mathbf{x}, \mathbf{u}\right) d t d \mathbf{x} d \mathbf{u} \rightarrow \int_{\mathcal{Q}^{\prime}} \int_{0}^{2 \pi / \lambda} \bar{G}(t, \tau, \mathbf{x}, \mathbf{u}) \varphi(t, \tau, \mathbf{x}, \mathbf{u}) d \tau d t d \mathbf{x} d \mathbf{v}
$$

On the other hand,

$$
\begin{aligned}
\int_{\mathcal{Q}^{\prime}} g^{\varepsilon}(t, \mathbf{x}, \mathbf{u}) \varphi\left(t, \frac{t}{\varepsilon^{2}}, \mathbf{x}, \mathbf{u}\right) d t d \mathbf{x} d \mathbf{u} & =\int_{\mathcal{Q}} g^{\varepsilon}\left(t, \mathbf{x}, \mathbf{r}\left(\mathbf{v}, \lambda \frac{t}{\varepsilon^{2}}\right)\right) \varphi\left(t, \frac{t}{\varepsilon^{2}}, \mathbf{x}, \mathbf{r}\left(\mathbf{v}, \lambda \frac{t}{\varepsilon^{2}}\right)\right) d t d \mathbf{x} d \mathbf{v} \\
& =\int_{\mathcal{Q}} f^{\varepsilon}(t, \mathbf{x}, \mathbf{v}) \varphi\left(t, \frac{t}{\varepsilon^{2}}, \mathbf{x}, \mathbf{r}\left(\mathbf{v}, \lambda \frac{t}{\varepsilon^{2}}\right)\right) d t d \mathbf{x} d \mathbf{v}
\end{aligned}
$$

which converges to

$$
\begin{gathered}
\int_{\mathcal{Q}} \int_{0}^{2 \pi / \lambda} F(t, \tau, \mathbf{x}, \mathbf{v}) \varphi(t, \tau, \mathbf{x}, \mathbf{r}(\mathbf{v}, \lambda \tau)) d \tau d t d \mathbf{x} d \mathbf{v}= \\
\int_{\mathcal{Q}} \int_{0}^{2 \pi / \lambda} G(t, \mathbf{x}, \mathbf{r}(\mathbf{v}, \lambda \tau)) \varphi(t, \tau, \mathbf{x}, \mathbf{r}(\mathbf{v}, \lambda \tau)) d \tau d t d \mathbf{x} d \mathbf{v}= \\
\int_{\mathcal{Q}^{\prime}} \int_{0}^{2 \pi / \lambda} G(t, \mathbf{x}, \mathbf{u}) \varphi(t, \tau, \mathbf{x}, \mathbf{u}) d \tau d t d \mathbf{x} d \mathbf{u} .
\end{gathered}
$$

Thus, we may conclude

$$
\bar{G}(t, \tau, \mathbf{x}, \mathbf{u})=G(t, \mathbf{x}, \mathbf{u}),
$$

which implies that $G$ is the two scale limit of $g^{\varepsilon}$. The first part of the Lemma is proved. Moreover

$$
\begin{aligned}
g(t, \tau, \mathbf{u}) & \left.=\int_{0}^{2 \pi / \lambda} \bar{G}(t, \tau, \mathbf{x}, \mathbf{u})\right) d \tau \\
& =\int_{0}^{2 \pi / \lambda} G(t, \mathbf{x}, \mathbf{u}) d \tau \\
& =\frac{2 \pi}{\lambda} G(t, \mathbf{x}, \mathbf{u})
\end{aligned}
$$

prooving the second part of Lemma 3.1.

Concerning the computation of the term containing the $\frac{1}{\varepsilon}$ factor in (3.4) we have:

Lemma 3.2 Denoting by $G(t, \mathbf{x}, \mathbf{u})$ the two scale limit of $g^{\varepsilon}$, which is also the function linked to the two scale limit $F$ of $f^{\varepsilon}$ by (2.5), the following formula holds true for all test functions $\varphi(t, \mathbf{x}, \mathbf{u})$ :

$\operatorname{RR} n^{\circ} 3428$

$$
\begin{aligned}
& \lim _{\varepsilon \rightarrow 0}\left\{\int _ { \mathcal { Q } ^ { \prime } } g ^ { \varepsilon } ( t , \mathbf { x } , \mathbf { u } ) \frac { 1 } { \varepsilon } \left[\left(u_{2} \cos \lambda \frac{t}{\varepsilon^{2}}+u_{3} \sin \lambda \frac{t}{\varepsilon^{2}}\right) \frac{\partial \varphi}{\partial x_{2}}(t, \mathbf{x}, \mathbf{u})\right.\right. \\
& \quad+\left(-u_{2} \sin \lambda \frac{t}{\varepsilon^{2}}+u_{3} \cos \lambda \frac{t}{\varepsilon^{2}}\right) \frac{\partial \varphi}{\partial x_{3}}(t, \mathbf{x}, \mathbf{u}) \\
& \quad+\lambda\left(\left(-\partial_{x_{2}} p\right) \cos \lambda \frac{t}{\varepsilon^{2}}+\left(\partial_{x_{3}} p\right) \sin \lambda \frac{t}{\varepsilon^{2}}\right) \frac{\partial \varphi}{\partial u_{2}}(t, \mathbf{x}, \mathbf{u})
\end{aligned}
$$




$$
\begin{aligned}
+\lambda\left(\left(-\partial_{x_{2}} p\right) \sin \lambda \frac{t}{\varepsilon^{2}}-\right. & \left.\left.\left(\partial_{x_{3}} p\right) \cos \lambda \frac{t}{\varepsilon^{2}}\right) \frac{\partial \varphi}{\partial u_{3}}(t, \mathbf{x}, \mathbf{u}) d \tau d t d \mathbf{x} d \mathbf{u}\right\}= \\
& -\frac{2 \pi}{\lambda} \int_{\mathcal{Q}^{\prime}} G(t, \mathbf{x}, \mathbf{u})\left[\partial_{x_{3}} p \frac{\partial \varphi}{\partial x_{2}}-\partial_{x_{2}} p \frac{\partial \varphi}{\partial x_{3}}\right. \\
+ & \left.\frac{1}{2}\left(\partial_{x_{2}}^{2} p+\partial_{x_{3}}^{2} p\right)\left(-u_{3} \frac{\partial \varphi}{\partial u_{2}}+u_{2} \frac{\partial \varphi}{\partial u_{3}}\right)\right] d t d \mathbf{x} d \mathbf{u} .
\end{aligned}
$$

Proof. As in the singular perturbation theory, see [7], we are looking for the limit of the inner product of $g^{\varepsilon}$ by a function which is not bounded but which converges to 0 in the sense of distributions. Indeed since

$$
\frac{1}{\varepsilon} \cos \lambda \frac{t}{\varepsilon^{2}}=\frac{d}{d t}\left(\frac{\varepsilon}{\lambda} \sin \lambda \frac{t}{\varepsilon^{2}}\right), \frac{1}{\varepsilon} \sin \lambda \frac{t}{\varepsilon^{2}}=\frac{d}{d t}\left(-\frac{\varepsilon}{\lambda} \cos \lambda \frac{t}{\varepsilon^{2}}\right) .
$$

those functions converges to 0 in the sense of distributions. Using this Remark, we develop a method which is in the same spirit as the one used by Cioranescu and Murat $[2,3]$.

For any $\varphi$, we build an oscillating test function $\alpha$ whose $\tau$-derivative when applied in $\tau=$ $\frac{t}{\varepsilon^{2}}$ gives $\frac{1}{\varepsilon}\left[\left(u_{2} \cos \lambda \frac{t}{\varepsilon^{2}}+u_{3} \sin \lambda \frac{t}{\varepsilon^{2}}\right) \frac{\partial \varphi}{\partial x_{2}}+\left(-u_{2} \sin \lambda \frac{t}{\varepsilon^{2}}+u_{3} \cos \lambda \frac{t}{\varepsilon^{2}}\right) \frac{\partial \varphi}{\partial x_{3}}+\lambda\left(\left(-\partial_{x_{2}} p\right) \cos \lambda \frac{t}{\varepsilon^{2}}+\right.\right.$ $\left.\left(\partial_{x_{3}} p\right) \sin \lambda \frac{t}{\varepsilon^{2}}\right) \frac{\partial \varphi}{\partial u_{2}}+\lambda\left(\left(-\partial_{x_{2}} p\right) \sin \lambda \frac{t}{\varepsilon^{2}}-\left(\partial_{x_{3}} p\right) \cos \lambda \frac{t}{\varepsilon^{2}}\right) \frac{\partial \varphi}{\partial u_{3}}$. Choosing the function $\alpha$ as a test function in the weak formulation (2.1) gives first an expression of the limit we want to compute in term of $\alpha$. Then using the expression of the function $\alpha$, an easy but fastidious integration leads to the result.

For any regular $\varphi(t, \mathbf{x}, \mathbf{u})$, we define

$$
\begin{aligned}
& \alpha(t, \tau, \mathbf{x}, \mathbf{u})=\frac{1}{\lambda}\left(u_{2} \sin \lambda \tau-u_{3} \cos \lambda \tau\right) \frac{\partial \varphi}{\partial x_{2}}(t, \mathbf{x}, \mathbf{u}) \\
& +\frac{1}{\lambda}\left(u_{2} \cos \lambda \tau+u_{3} \sin \lambda \tau\right) \frac{\partial \varphi}{\partial x_{3}}(t, \mathbf{x}, \mathbf{u}) \\
& +\left(\left(-\partial_{x_{2}} p\right) \sin \lambda \tau-\left(\partial_{x_{3}} p\right) \cos \lambda \tau\right) \frac{\partial \varphi}{\partial u_{2}}(t, \mathbf{x}, \mathbf{u}) \\
& +\left(\left(\partial_{x_{2}} p\right) \cos \lambda \tau-\left(\partial_{x_{3}} p\right) \sin \lambda \tau\right) \frac{\partial \varphi}{\partial u_{3}}(t, \mathbf{x}, \mathbf{u}),
\end{aligned}
$$

and we consider

$$
\beta(t, \tau, \mathbf{x}, \mathbf{v})=\alpha(t, \tau, \mathbf{x}, \mathbf{r}(\mathbf{v}, \lambda \tau))
$$

Applying the contraint operator to $\beta$, we get

$$
\begin{aligned}
& \frac{\partial \beta}{\partial \tau}+\lambda(\mathbf{v} \times \mathcal{M}) \cdot \nabla_{v} \beta= \\
& \quad \frac{\partial \alpha}{\partial \tau}+\lambda \frac{\partial \mathbf{r}}{\partial \tau}(\mathbf{v}, \lambda \tau) \cdot \nabla_{u} \alpha+\lambda(\mathbf{v} \times \mathcal{M}) \cdot \nabla_{v} \alpha=\left(\frac{\partial \alpha}{\partial \tau}\right)(t, \tau, \mathbf{x}, \mathbf{r}(\mathbf{v}, \lambda \tau))
\end{aligned}
$$

and the computation of $\frac{\partial \alpha}{\partial \tau}$ gives 


$$
\begin{aligned}
& \left(\frac{\partial \alpha}{\partial \tau}\right)(t, \tau, \mathbf{x}, \mathbf{u})=\left(u_{2} \cos \lambda \tau+u_{3} \sin \lambda \tau\right) \frac{\partial \varphi}{\partial x_{2}}(t, \mathbf{x}, \mathbf{u}) \\
& +\left(-u_{2} \sin \lambda \tau+u_{3} \cos \lambda \tau\right) \frac{\partial \varphi}{\partial x_{3}}(t, \mathbf{x}, \mathbf{u}) \\
& +\lambda\left(\left(-\partial_{x_{2}} p\right) \cos \lambda \tau+\left(\partial_{x_{3}} p\right) \sin \lambda \tau\right) \frac{\partial \varphi}{\partial u_{2}}(t, \mathbf{x}, \mathbf{u}) \\
& +\lambda\left(\left(-\partial_{x_{2}} p\right) \sin \lambda \tau-\left(\partial_{x_{3}} p\right) \cos \lambda \tau\right) \frac{\partial \varphi}{\partial u_{3}}(t, \mathbf{x}, \mathbf{u})
\end{aligned}
$$

Beside this, easy computations give

$$
\begin{gathered}
\left(\frac{\partial \beta}{\partial t}\right)^{\varepsilon}=\left(\frac{\partial \alpha}{\partial t}\right)\left(t, \frac{t}{\varepsilon^{2}}, \mathbf{x}, \mathbf{r}\left(\mathbf{v}, \lambda \frac{t}{\varepsilon^{2}}\right)\right) \\
\left(\frac{\partial \beta}{\partial x_{i}}\right)^{\varepsilon}=\left(\frac{\partial \alpha}{\partial x_{i}}\right)\left(t, \frac{t}{\varepsilon^{2}}, \mathbf{x}, \mathbf{r}\left(\mathbf{v}, \lambda \frac{t}{\varepsilon^{2}}\right)\right) \\
\left(\frac{\partial \beta}{\partial v_{2}}\right)^{\varepsilon}=\cos \lambda \frac{t}{\varepsilon^{2}}\left(\frac{\partial \alpha}{\partial u_{2}}\right)\left(t, \frac{t}{\varepsilon^{2}}, \mathbf{x}, \mathbf{r}\left(\mathbf{v}, \lambda \frac{t}{\varepsilon^{2}}\right)\right)+\sin \lambda \frac{t}{\varepsilon^{2}}\left(\frac{\partial \alpha}{\partial u_{3}}\right)\left(t, \frac{t}{\varepsilon^{2}}, \mathbf{x}, \mathbf{r}\left(\mathbf{v}, \lambda \frac{t}{\varepsilon^{2}}\right)\right) \\
\left(\frac{\partial \beta}{\partial v_{3}}\right)^{\varepsilon}=-\sin \lambda \frac{t}{\varepsilon^{2}}\left(\frac{\partial \alpha}{\partial u_{2}}\right)\left(t, \frac{t}{\varepsilon^{2}}, \mathbf{x}, \mathbf{r}\left(\mathbf{v}, \lambda \frac{t}{\varepsilon^{2}}\right)\right)+\cos \lambda \frac{t}{\varepsilon^{2}}\left(\frac{\partial \alpha}{\partial u_{3}}\right)\left(t, \frac{t}{\varepsilon^{2}}, \mathbf{x}, \mathbf{r}\left(\mathbf{v}, \lambda \frac{t}{\varepsilon^{2}}\right)\right) .
\end{gathered}
$$

Having those formulae at hand, we use test functions

$$
\varepsilon(\beta)^{\varepsilon}=\varepsilon \beta\left(t, \frac{t}{\varepsilon^{2}}, \mathbf{x}, \mathbf{v}\right),
$$

in the weak formulation (2.1). We get

$$
\begin{aligned}
\int_{\mathcal{Q}} f^{\varepsilon}\left[\varepsilon\left(\frac{\partial \beta}{\partial t}\right)^{\varepsilon}+\frac{1}{\varepsilon}\left(\frac{\partial \beta}{\partial \tau}\right)^{\varepsilon}+\mathbf{v} \cdot\left(\nabla_{x} \beta\right)^{\varepsilon}+\lambda\left(-\nabla_{x} p+\mathbf{v}\right.\right. & \left.\left.\times \frac{\mathcal{M}}{\varepsilon}\right) \cdot\left(\nabla_{v} \beta\right)^{\varepsilon}\right] d t d \mathbf{x} d \mathbf{v} \\
& =-\varepsilon \int_{\Omega} f_{0} \beta(0,0, ., .) d \mathbf{x} d \mathbf{v}
\end{aligned}
$$

Now, using formula (3.10), the expression of $f^{\varepsilon}$ in term of $g^{\varepsilon}$ and the expressions of the derivatives of $\beta$ (3.11)-(3.15), equation (3.17) becomes

$$
\begin{aligned}
\int_{\mathcal{Q}} g^{\varepsilon}\left(t, \mathbf{x}, \mathbf{r}\left(\mathbf{v}, \lambda \frac{t}{\varepsilon^{2}}\right)\right)\left[\varepsilon\left(\frac{\partial \alpha}{\partial t}\right)\left(t, \frac{t}{\varepsilon^{2}}, \mathbf{x}, \mathbf{r}\left(\mathbf{v}, \lambda \frac{t}{\varepsilon^{2}}\right)\right)\right. \\
+\frac{1}{\varepsilon}\left(\frac{\partial \alpha}{\partial \tau}\right)\left(t, \frac{t}{\varepsilon^{2}}, \mathbf{x}, \mathbf{r}\left(\mathbf{v}, \lambda \frac{t}{\varepsilon^{2}}\right)\right)+\mathbf{v} \cdot\left(\nabla_{x} \alpha\right)\left(t, \frac{t}{\varepsilon^{2}}, \mathbf{x}, \mathbf{r}\left(\mathbf{v}, \lambda \frac{t}{\varepsilon^{2}}\right)\right) \\
\left.-\lambda\left(\begin{array}{l}
\left(\partial_{x_{2}} p\right) \cos \lambda \frac{t}{\varepsilon^{2}}-\left(\partial_{x_{3}} p\right) \sin \lambda \frac{t}{\varepsilon^{2}} \\
\left(\partial_{x_{2}} p\right) \sin \lambda \frac{t}{\varepsilon^{2}}+\left(\partial_{x_{3}} p\right) \cos \lambda \frac{t}{\varepsilon^{2}}
\end{array}\right) \cdot\left(\nabla_{u} \alpha\right)\left(t, \frac{t}{\varepsilon^{2}}, \mathbf{x}, \mathbf{r}\left(\mathbf{v}, \lambda \frac{t}{\varepsilon^{2}}\right)\right)\right] d t d \mathbf{x} d \mathbf{v} \\
=-\varepsilon \int_{\Omega} f_{0} \alpha(0,0, ., .) d \mathbf{x} d \mathbf{v}
\end{aligned}
$$

$\operatorname{RR} n^{\circ} 3428$ 
In equation (3.18) we make the change of variable (3.2), it gives

$$
\begin{aligned}
& \int_{\mathcal{Q}^{\prime}} g^{\varepsilon}(t, \mathbf{x}, \mathbf{u})\left[\varepsilon\left(\frac{\partial \alpha}{\partial t}\right)\left(t, \frac{t}{\varepsilon^{2}}, \mathbf{x}, \mathbf{u}\right)\right. \\
& +\frac{1}{\varepsilon}\left(\frac{\partial \alpha}{\partial \tau}\right)\left(t, \frac{t}{\varepsilon^{2}}, \mathbf{x}, \mathbf{u}\right)+\mathbf{r}\left(\mathbf{u},-\lambda \frac{t}{\varepsilon^{2}}\right) \cdot\left(\nabla_{x} \alpha\right)\left(t, \frac{t}{\varepsilon^{2}}, \mathbf{x}, \mathbf{u}\right) \\
& \left.-\lambda\left(\begin{array}{l}
\left(\partial_{x_{2}} p\right) \cos \lambda \frac{t}{\varepsilon^{2}}-\left(\partial_{x_{3}} p\right) \sin \lambda \frac{t}{\varepsilon^{2}} \\
\left(\partial_{x_{2}} p\right) \sin \lambda \frac{t}{\varepsilon^{2}}+\left(\partial_{x_{3}} p\right) \cos \lambda \frac{t}{\varepsilon^{2}}
\end{array}\right) \cdot\left(\nabla_{u} \alpha\right)\left(t, \frac{t}{\varepsilon^{2}}, \mathbf{x}, \mathbf{u}\right)\right] d t d \mathbf{x} d \mathbf{u} \\
& =-\varepsilon \int_{\Omega} f_{0} \alpha(0,0, ., .) d \mathbf{x} d \mathbf{u}
\end{aligned}
$$

which, passing to the limit in $\varepsilon$, using Lemma 3.1 gives

$$
\begin{aligned}
\lim _{\varepsilon \rightarrow 0}\left\{\int_{\mathcal{Q}^{\prime}} g^{\varepsilon}(t, \mathbf{x}, \mathbf{u}) \frac{1}{\varepsilon}\left(\frac{\partial \alpha}{\partial \tau}\right)\left(t, \frac{t}{\varepsilon^{2}}, \mathbf{x}, \mathbf{u}\right) d t d \mathbf{x} d \mathbf{u}\right\}= \\
-\int_{\mathcal{Q}^{\prime}} G \int_{0}^{2 \pi / \lambda}\left[\left(\begin{array}{c}
u_{2} \cos \lambda \tau+u_{3} \sin \lambda \tau \\
-u_{2} \sin \lambda \tau+u_{3} \cos \lambda \tau
\end{array}\right) \cdot\left(\nabla_{x} \alpha\right)(t, \tau, \mathbf{x}, \mathbf{u})\right. \\
\left.\quad-\lambda\left(\begin{array}{c}
\left(\partial_{x_{2}} p\right) \cos \lambda \tau-\left(\partial_{x_{3}} p\right) \sin \lambda \tau \\
\left(\partial_{x_{2}} p\right) \sin \lambda \tau+\left(\partial_{x_{3}} p\right) \cos \lambda \tau
\end{array}\right) \cdot\left(\nabla_{u} \alpha\right)(t, \tau, \mathbf{x}, \mathbf{u})\right] d \tau d t d \mathbf{x} d \mathbf{u},
\end{aligned}
$$

and

$$
\begin{aligned}
& \int_{\mathcal{Q}^{\prime}} g^{\varepsilon}(t, \mathbf{x}, \mathbf{u}) \frac{1}{\varepsilon}\left(\frac{\partial \alpha}{\partial \tau}\right)\left(t, \frac{t}{\varepsilon^{2}}, \mathbf{x}, \mathbf{u}\right) d t d \mathbf{x} d \mathbf{u}= \\
& \quad \int_{\mathcal{Q}^{\prime}} g^{\varepsilon}(t, \mathbf{x}, \mathbf{u}) \frac{1}{\varepsilon}\left[\left(u_{2} \cos \lambda \frac{t}{\varepsilon^{2}}+u_{3} \sin \lambda \frac{t}{\varepsilon^{2}}\right) \frac{\partial \varphi}{\partial x_{2}}(t, \mathbf{x}, \mathbf{u})\right. \\
& \quad+\left(-u_{2} \sin \lambda \frac{t}{\varepsilon^{2}}+u_{3} \cos \lambda \frac{t}{\varepsilon^{2}}\right) \frac{\partial \varphi}{\partial x_{3}}(t, \mathbf{x}, \mathbf{u}) \\
& \quad+\lambda\left(\left(-\partial_{x_{2}} p\right) \cos \lambda \frac{t}{\varepsilon^{2}}+\left(\partial_{x_{3}} p\right) \sin \lambda \frac{t}{\varepsilon^{2}}\right) \frac{\partial \varphi}{\partial u_{2}}(t, \mathbf{x}, \mathbf{u}) \\
& \left.\quad+\lambda\left(\left(-\partial_{x_{2}} p\right) \sin \lambda \frac{t}{\varepsilon^{2}}-\left(\partial_{x_{3}} p\right) \cos \lambda \frac{t}{\varepsilon^{2}}\right) \frac{\partial \varphi}{\partial u_{3}}(t, \mathbf{x}, \mathbf{u})\right] d \tau d t d \mathbf{x} d \mathbf{u},
\end{aligned}
$$

is exactly the term whose limit we want to compute in (3.6).

At this level, formula (3.20) gives the seeked limit in terms of $\alpha$. Hence we now have to replace, in the right hand side of (3.20) $\alpha$ by its expression in term of $\varphi$ and to integrate in $\tau$. This step is straightforward but fastidious. The detailed computation yielding the following formula is given in the appendix.

We have

$$
\begin{array}{r}
\int_{0}^{2 \pi / \lambda}\left[\left(\begin{array}{c}
u_{2} \cos \lambda \tau+u_{3} \sin \lambda \tau \\
-u_{2} \sin \lambda \tau+u_{3} \cos \lambda \tau
\end{array}\right) \cdot\left(\nabla_{x} \alpha\right)-\lambda\left(\begin{array}{c}
\left(\partial_{x_{2}} p\right) \cos \lambda \tau-\left(\partial_{x_{3}} p\right) \sin \lambda \tau \\
\left(\partial_{x_{2}} p\right) \sin \lambda \tau+\left(\partial_{x_{3}} p\right) \cos \lambda \tau
\end{array}\right) \cdot\left(\nabla_{u} \alpha\right)\right] d \tau= \\
\left.\frac{2 \pi}{\lambda}\left(\partial_{x_{3}} p \frac{\partial \varphi}{\partial x_{2}}-\partial_{x_{2}} p \frac{\partial \varphi}{\partial x_{3}}\right)+\frac{\pi}{\lambda}\left(\partial_{x_{2}}^{2} p+\partial_{x_{3}}^{2} p\right)\left(-u_{3} \frac{\partial \varphi}{\partial u_{2}}+u_{2} \frac{\partial \varphi}{\partial u_{3}}\right)\right] .
\end{array}
$$


Using then (3.22) in (3.20) gives the Lemma.

Since Lemma 3.1 and 3.2 give the two scale- and weak - * - limits of $g^{\varepsilon}$ and the limit of the term containing the $\frac{1}{\varepsilon}$ in $(3.4)$, we can pass to the limit and obtain

$$
\begin{array}{r}
\frac{2 \pi}{\lambda} \int_{\mathcal{Q}^{\prime}} G\left[\frac{\partial \varphi}{\partial t}-\partial_{x_{3}} p \frac{\partial \varphi}{\partial x_{2}}+\partial_{x_{2}} p \frac{\partial \varphi}{\partial x_{3}}+\frac{1}{2}\left(\partial_{x_{2}}^{2} p+\partial_{x_{3}}^{2} p\right)\right. \\
\left.\left(u_{3} \frac{\partial \varphi}{\partial u_{2}}-u_{2} \frac{\partial \varphi}{\partial u_{3}}\right)\right] d t d \mathbf{x} d \mathbf{u} \\
=-\int_{\Omega} f_{0} \varphi(0, \mathbf{x}, \mathbf{v}) d \mathbf{x} d \mathbf{u}
\end{array}
$$

for all $\varphi(t, \mathbf{x}, \mathbf{v})$ regular and with compact support, which is the weak formulation of the equation for $G$ we are seeking. Thus noticing

$$
-\partial_{x_{3}} p \frac{\partial \varphi}{\partial x_{2}}+\partial_{x_{2}} p \frac{\partial \varphi}{\partial x_{3}}=\left(-\nabla_{x} p \times \mathcal{M}\right) \cdot \nabla_{x} \varphi
$$

and

$$
u_{3} \frac{\partial \varphi}{\partial u_{2}}-u_{2} \frac{\partial \varphi}{\partial u_{3}}=(\mathbf{u} \times \mathcal{M}) \cdot \nabla_{u} \varphi
$$

and denoting

$$
\Delta p=\partial_{x_{2}}^{2} p+\partial_{x_{3}}^{2} p
$$

we have proved the

TheOREM 3.3 Under assumptions (1.2), the sequence $\left(f^{\varepsilon}(t, \mathbf{x}, \mathbf{v})\right)_{\varepsilon}$ of solutions of (1.1) two-scale converges to a profile $F(t, \tau, \mathbf{x}, \mathbf{v}) \in L^{\infty}\left(0, T ; L_{2 \pi / \lambda}^{\infty}\left(I R_{\tau} ; L^{2}(\Omega)\right)\right)$ satisfying

$$
F(t, \tau, \mathbf{x}, \mathbf{v})=G(t, \mathbf{x}, \mathbf{r}(\mathbf{v}, \lambda \tau))
$$

where $\mathbf{r}(\mathbf{v}, \tau)$ is given by (2.4) and where $G \equiv G(t, \mathbf{x}, \mathbf{u}) \in L^{\infty}\left(0, T ; L^{2}\left(\mathbb{R}_{x}^{2} \times \mathbb{R}_{u}^{2}\right)\right)$ is the unique solution of

$$
\left\{\begin{array}{l}
\frac{\partial G}{\partial t}+\left(-\nabla_{x} p \times \mathcal{M}\right) \cdot \nabla_{x} G+\frac{1}{2}(\Delta p(\mathbf{u} \times \mathcal{M})) \cdot \nabla_{u} G=0 \\
G_{\mid t=0}=\frac{\lambda}{2 \pi} f_{0}(\mathbf{x}, \mathbf{r}(\mathbf{v}, \lambda \tau)) .
\end{array}\right.
$$

\section{Equation for $f$, proof of the Theorems}

Using the relation (1.6) linking $f$ and $F$, we have

$$
f=\int_{0}^{2 \pi / \lambda} F(., \tau, ., .) d \tau=\int_{0}^{2 \pi / \lambda} G(., ., \mathbf{r}(., \lambda \tau)) d \tau
$$

and noticing

$$
(\mathbf{r}(\mathbf{v}, \lambda \tau) \times \mathcal{M})) \cdot \nabla_{u} G(t, \mathbf{x}, \mathbf{r}(\mathbf{v}, \lambda \tau))=(\mathbf{v} \times \mathcal{M}) \cdot \nabla_{v}[G(t, \mathbf{x}, \mathbf{r}(\mathbf{v}, \lambda \tau))],
$$

$\mathrm{RR} \mathrm{n}^{\circ} 3428$ 
replacing $\mathbf{u}$ by $\mathbf{r}(\mathbf{v}, \lambda \tau)$ in (3.28) gives

$$
\left\{\begin{aligned}
\frac{\partial}{\partial t}[G(t, \mathbf{x}, \mathbf{r}(\mathbf{v}, \lambda \tau))]+\left(-\nabla_{x} p \times \mathcal{M}\right) \cdot \nabla_{x}[G(t, \mathbf{x}, \mathbf{r}(\mathbf{v}, \lambda \tau))] & \\
+ & \frac{1}{2}(\Delta p(\mathbf{v} \times \mathcal{M})) \cdot \nabla_{v}[G(t, \mathbf{x}, \mathbf{r}(\mathbf{v}, \lambda \tau))]=0 \\
G(0, \mathbf{x}, \mathbf{r}(\mathbf{v}, \lambda \tau)) & =\frac{\lambda}{2 \pi} f_{0}(\mathbf{x}, \mathbf{r}(\mathbf{v}, \lambda \tau)) .
\end{aligned}\right.
$$

Integrating (4.3) in $\tau$ from 0 to $2 \pi / \lambda$ finally gives equation (1.8) and achieve the proof of Theorem 1.1.

Noticing

$$
\nabla_{v} \cdot(\Delta p(\mathbf{v} \times \mathcal{M}))=0
$$

and

$$
\frac{1}{2 \pi} \int_{0}^{2 \pi} \int_{\mathbb{R}_{v}^{2}} f_{0}(\mathbf{x}, \mathbf{r}(\mathbf{v}, \tau)) d \mathbf{v} d \tau=\int_{\mathbb{R}_{v}^{2}} f_{0}(\mathbf{x}, \mathbf{v}) d \mathbf{v}
$$

the proof of Theorem 1.2 is straightforward integrating (1.8) in $\mathbf{v}$ and summing in $s$. 


\section{A Appendix: Computation yielding (3.22)}

In this appendix, we gives the fastidious computation yielding formula (3.22). Using the definition of $\alpha$ in term of $\varphi$, we have

$$
\begin{aligned}
& \int_{0}^{2 \pi / \lambda}\left[\left(\begin{array}{c}
u_{2} \cos \lambda \tau+u_{3} \sin \lambda \tau \\
-u_{2} \sin \lambda \tau+u_{3} \cos \lambda \tau
\end{array}\right) \cdot\left(\nabla_{x} \alpha\right)-\lambda\left(\begin{array}{c}
\left(\partial_{x_{2}} p\right) \cos \lambda \tau-\left(\partial_{x_{3}} p\right) \sin \lambda \tau \\
\left(\partial_{x_{2}} p\right) \sin \lambda \tau+\left(\partial_{x_{3}} p\right) \cos \lambda \tau
\end{array}\right) \cdot\left(\nabla_{u} \alpha\right)\right] d \tau= \\
& \int_{0}^{2 \pi / \lambda}\left[( u _ { 2 } \operatorname { c o s } \lambda \tau + u _ { 3 } \operatorname { s i n } \lambda \tau ) \left\{\frac{1}{\lambda}\left(u_{2} \sin \lambda \tau-u_{3} \cos \lambda \tau\right)\left(\frac{\partial^{2} \varphi}{\partial x_{2}^{2}} x_{2}\right)+\right.\right. \\
& \frac{1}{\lambda}\left(u_{2} \cos \lambda \tau+u_{3} \sin \lambda \tau\right)\left(\frac{\partial^{2} \varphi}{\partial x_{2}^{2}} x_{3}\right) \\
& +\left(\left(-\partial_{x_{2} x_{2}}^{2} p\right) \sin \lambda \tau-\left(\partial_{x_{2} x_{3}}^{2} p\right) \cos \lambda \tau\right)\left(\frac{\partial \varphi}{\partial u_{2}}\right)+\left(\left(-\partial_{x_{2}} p\right) \sin \lambda \tau-\left(\partial_{x_{3}} p\right) \cos \lambda \tau\right)\left(\frac{\partial^{2} \varphi}{\partial x_{2}^{2}} u_{2}\right) \\
& \left.+\left(\left(\partial_{x_{2} x_{2}}^{2} p\right) \cos \lambda \tau-\left(\partial_{x_{2} x_{3}}^{2} p\right) \sin \lambda \tau\right)\left(\frac{\partial \varphi}{\partial u_{3}}\right)+\left(\left(\partial_{x_{2}} p\right) \cos \lambda \tau-\left(\partial_{x_{3}} p\right) \sin \lambda \tau\right)\left(\frac{\partial^{2} \varphi}{\partial x_{2}^{2}} u_{3}\right)\right\} \\
& +\left(-u_{2} \sin \lambda \tau+u_{3} \cos \lambda \tau\right)\left\{\frac{1}{\lambda}\left(u_{2} \sin \lambda \tau-u_{3} \cos \lambda \tau\right)\left(\frac{\partial^{2} \varphi}{\partial x_{2}^{2}} x_{3}\right)+\frac{1}{\lambda}\left(u_{2} \cos \lambda \tau+u_{3} \sin \lambda \tau\right)\left(\frac{\partial^{2} \varphi}{\partial x_{3}^{2}} x_{3}\right)\right. \\
& +\left(\left(-\partial_{x_{2} x_{3}}^{2} p\right) \sin \lambda \tau-\left(\partial_{x_{3} x_{3}}^{2} p\right) \cos \lambda \tau\right)\left(\frac{\partial \varphi}{\partial u_{2}}\right)+\left(\left(-\partial_{x_{2}} p\right) \sin \lambda \tau-\left(\partial_{x_{3}} p\right) \cos \lambda \tau\right)\left(\frac{\partial^{2} \varphi}{\partial x_{3}^{2}} u_{2}\right) \\
& \left.+\left(\left(\partial_{x_{2} x_{3}}^{2} p\right) \cos \lambda \tau-\left(\partial_{x_{3} x_{3}}^{2} p\right) \sin \lambda \tau\right)\left(\frac{\partial \varphi}{\partial u_{3}}\right)+\left(\left(\partial_{x_{2}} p\right) \cos \lambda \tau-\left(\partial_{x_{3}} p\right) \sin \lambda \tau\right)\left(\frac{\partial^{2} \varphi}{\partial x_{3}^{2}} u_{3}\right)\right\} \\
& +\left(\left(-\partial_{x_{2}} p\right) \cos \lambda \tau+\left(\partial_{x_{3}} p\right) \sin \lambda \tau\right)\left\{\sin \lambda \tau\left(\frac{\partial \varphi}{\partial x_{2}}\right)+\cos \lambda \tau\left(\frac{\partial \varphi}{\partial x_{3}}\right)\right. \\
& +\left(u_{2} \sin \lambda \tau-u_{3} \cos \lambda \tau\right)\left(\frac{\partial^{2} \varphi}{\partial x_{2}^{2}} u_{2}\right)+\left(u_{2} \cos \lambda \tau+u_{3} \sin \lambda \tau\right)\left(\frac{\partial^{2} \varphi}{\partial x_{3}^{2}} u_{2}\right) \\
& \left.+\lambda\left(\left(-\partial_{x_{2}} p\right) \sin \lambda \tau-\left(\partial_{x_{3}} p\right) \cos \lambda \tau\right)\left(\frac{\partial^{2} \varphi}{\partial u_{2}^{2}} u_{2}\right)+\lambda\left(\left(\partial_{x_{2}} p\right) \cos \lambda \tau-\left(\partial_{x_{3}} p\right) \sin \lambda \tau\right)\left(\frac{\partial^{2} \varphi}{\partial u_{2}^{2}} u_{3}\right)\right\} \\
& +\left(\left(-\partial_{x_{2}} p\right) \sin \lambda \tau-\left(\partial_{x_{3}} p\right) \cos \lambda \tau\right)\left\{-\cos \lambda \tau\left(\frac{\partial \varphi}{\partial x_{2}}\right)+\sin \lambda \tau\left(\frac{\partial \varphi}{\partial x_{3}}\right)\right. \\
& \left(u_{2} \sin \lambda \tau-u_{3} \cos \lambda \tau\right)\left(\frac{\partial^{2} \varphi}{\partial x_{2}^{2}} u_{3}\right)+\left(u_{2} \cos \lambda \tau+u_{3} \sin \lambda \tau\right)\left(\frac{\partial^{2} \varphi}{\partial x_{3}^{2}} u_{3}\right) \\
& \left.\left.+\lambda\left(\left(-\partial_{x_{2}} p\right) \sin \lambda \tau-\left(\partial_{x_{3}} p\right) \cos \lambda \tau\right)\left(\frac{\partial^{2} \varphi}{\partial u_{2}^{2}} u_{3}\right)+\lambda\left(\left(\partial_{x_{2}} p\right) \cos \lambda \tau-\left(\partial_{x_{3}} p\right) \sin \lambda \tau\right)\left(\frac{\partial^{2} \varphi}{\partial u_{3}^{2}} u_{3}\right)\right\}\right] d \tau .
\end{aligned}
$$

In order to provide the computations in (A.1) we first use (to reduce the number of terms) $\int_{0}^{2 \pi / \lambda} \sin \lambda \tau \cos \lambda \tau d \tau=0$. We then get 


$$
\begin{aligned}
& \int_{0}^{2 \pi / \lambda}\left[\left(\frac{\partial \varphi}{\partial x_{2}}\right)\left(\cos ^{2} \lambda \tau+\sin ^{2} \lambda \tau\right)\left(\partial_{x_{3}} p\right)-\left(\frac{\partial \varphi}{\partial x_{3}}\right)\left(\cos ^{2} \lambda \tau+\sin ^{2} \lambda \tau\right)\left(\partial_{x_{2}} p\right)\right. \\
& +\left(\frac{\partial \varphi}{\partial u_{2}}\right)\left(-u_{2}\left(\cos ^{2} \lambda \tau-\sin ^{2} \lambda \tau\right)\left(\partial_{x_{2} x_{3}}^{2} p\right)-u_{3} \sin ^{2} \lambda \tau\left(\partial_{x_{2} x_{2}}^{2} p\right)-u_{3} \cos ^{2} \lambda \tau\left(\partial_{x_{3} x_{3}}^{2} p\right)\right) \\
& +\left(\frac{\partial \varphi}{\partial u_{3}}\right)\left(u_{3}\left(\cos ^{2} \lambda \tau-\sin ^{2} \lambda \tau\right)\left(\partial_{x_{2} x_{3}}^{2} p\right)+u_{2} \cos ^{2} \lambda \tau\left(\partial_{x_{2} x_{2}}^{2} p\right)+u_{2} \sin ^{2} \lambda \tau\left(\partial_{x_{3} x_{3}}^{2} p\right)\right) \\
& +\frac{1}{\lambda}\left(\frac{\partial^{2} \varphi}{\partial x_{2}^{2}} x_{2}\right)\left(-u_{2} u_{3}\left(\cos ^{2} \lambda \tau-\sin ^{2} \lambda \tau\right)\right)+\left(\frac{\partial^{2} \varphi}{\partial x_{3}^{2}} x_{3}\right)\left(u_{2} u_{3}\left(\cos ^{2} \lambda \tau-\sin ^{2} \lambda \tau\right)\right) \\
& +\frac{1}{\lambda}\left(\frac{\partial^{2} \varphi}{\partial x_{2}^{2}} x_{3}\right)\left(u_{2}^{2}\left(\cos ^{2} \lambda \tau-\sin ^{2} \lambda \tau\right)-u_{3}^{2}\left(\cos ^{2} \lambda \tau-\sin ^{2} \lambda \tau\right)\right) \\
& +\lambda\left(\frac{\partial^{2} \varphi}{\partial u_{2}^{2}} u_{2}\right)\left(\left(\partial_{x_{2}} p\right)\left(\partial_{x_{3}} p\right)\left(\cos ^{2} \lambda \tau-\sin ^{2} \lambda \tau\right)\right) \\
& +\lambda\left(\frac{\partial^{2} \varphi}{\partial u_{3}^{2}} u_{3}\right)\left(\left(\partial_{x_{2}} p\right)\left(\partial_{x_{3}} p\right)\left(\cos ^{2} \lambda \tau-\sin ^{2} \lambda \tau\right)\right) \\
& +\lambda\left(\frac{\partial^{2} \varphi}{\partial u_{2}^{2}} u_{3}\right)\left(-\left(\partial_{x_{2}} p\right)^{2}\left(\cos ^{2} \lambda \tau-\sin ^{2} \lambda \tau\right)+\left(\partial_{x_{3}} p\right)^{2}\left(\cos ^{2} \lambda \tau-\sin ^{2} \lambda \tau\right)\right) \\
& +\left(\frac{\partial^{2} \varphi}{\partial x_{2}^{2}} u_{2}\right)\left(-u_{2}\left(\partial_{x_{3}} p\right)\left(\cos ^{2} \lambda \tau-\sin ^{2} \lambda \tau\right)+u_{3}\left(\partial_{x_{2}} p\right)\left(\cos ^{2} \lambda \tau-\sin ^{2} \lambda \tau\right)\right) \\
& +\left(\frac{\partial^{2} \varphi}{\partial x_{2}^{2}} u_{3}\right)\left(u_{2}\left(\partial_{x_{2}} p\right)\left(\cos ^{2} \lambda \tau-\sin ^{2} \lambda \tau\right)+u_{3}\left(\partial_{x_{3}} p\right)\left(\cos ^{2} \lambda \tau-\sin ^{2} \lambda \tau\right)\right) \\
& +\left(\frac{\partial^{2} \varphi}{\partial x_{3}^{2}} u_{2}\right)\left(-u_{2}\left(\partial_{x_{2}} p\right)\left(\cos ^{2} \lambda \tau-\sin ^{2} \lambda \tau\right)-u_{3}\left(\partial_{x_{3}} p\right)\left(\cos ^{2} \lambda \tau-\sin ^{2} \lambda \tau\right)\right) \\
& \left.+\left(\frac{\partial^{2} \varphi}{\partial x_{3}^{2}} u_{3}\right)\left(-u_{2}\left(\partial_{x_{3}} p\right)\left(\cos ^{2} \lambda \tau-\sin ^{2} \lambda \tau\right)+u_{3}\left(\partial_{x_{2}} p\right)\left(\cos ^{2} \lambda \tau-\sin ^{2} \lambda \tau\right)\right)\right] d \tau
\end{aligned}
$$

Finally, using that $\int_{0}^{2 \pi / \lambda}\left(\cos ^{2} \lambda \tau+\sin ^{2} \lambda \tau\right) d \tau=2 \pi / \lambda, \int_{0}^{2 \pi / \lambda}\left(\cos ^{2} \lambda \tau-\sin ^{2} \lambda \tau\right) d \tau=0$, $\int_{0}^{2 \pi / \lambda} \cos ^{2} \lambda \tau d \tau=\pi / \lambda$, and $\int_{0}^{2 \pi / \lambda} \sin ^{2} \lambda \tau d \tau=\pi / \lambda$, (A.2) gives

$$
\frac{2 \pi}{\lambda}\left(\partial_{x_{3}} p \frac{\partial \varphi}{\partial x_{2}}-\partial_{x_{2}} p \frac{\partial \varphi}{\partial x_{3}}\right)+\frac{\pi}{\lambda}\left(\partial_{x_{2}}^{2} p+\partial_{x_{3}}^{2} p\right)\left(-u_{3} \frac{\partial \varphi}{\partial u_{2}}+u_{2} \frac{\partial \varphi}{\partial u_{3}}\right),
$$

which is the left hand side of (3.22). 


\section{References}

[1] G. Allaire. Homogeneization and Two-scale Convergence. SIAM J. Math. Anal., XXIII(6):1482-1518, 1992.

[2] D. Cioranescu and F. Murat. Un terme étrange venu d'ailleurs. In Research Note in Mathematics H. Brezis, J. L. Lions, editor, Nonlinear partial differential equations and their application, Collège de France Seminar, volume 2, pages 98-138. Pitman, London, 1982.

[3] D. Cioranescu and F. Murat. Un terme étrange venu d'ailleurs ii. In Research Note in Mathematics H. Brezis, J. L. Lions, editor, Nonlinear partial differential equations and their application, Collège de France Seminar, volume 3, pages 154-178. Pitman, London, 1982.

[4] D. H. E. Dubin, J. A. Krommes, C. Oberman, and W. W. Lee. Nonlinear gyrokinetic equations. Phys. Fluids, XXVI(12):3524-3535, 1983.

[5] E. Frénod and E. Sonnendrücker. Homogenization of the Vlasov equation and of the Vlasov-Poisson system with a strong external magnetic field. INRIA report, 3327, 1997.

[6] W. W. Lee. Gyrokinetic approach in particle simulation. Phys. Fluids, 26(2):556-562, 1983.

[7] J. L. Lions. Pertubations singulières dans les problemes aux limites et en controle optimal. Number 323 in Lecture notes in Mathematics. Springer-Verlag, 1973.

[8] Robert G. Littlejohn. Hamiltonian formulation of guiding center motion. Phys. Fluids, $24,1981$.

[9] G. N'Guetseng. A general convergence result for a functional related to the theory of homogeneization. SIAM J. Math. Anal., 20(3):608-623, 1989.

[10] Theodore G. Northrop. The guiding center approximation to charged particle motion. Ann. Phys., 15:79-101, 1961. 
Unit'e de recherche INRIA Lorraine, Technop`ole de Nancy-Brabois, Campus scientifique, 615 rue du Jardin Botanique, BP 101, 54600 VILLERS LÈS NANCY

Unit'e de recherche INRIA Rennes, Irisa, Campus universitaire de Beaulieu, 35042 RENNES Cedex

Unit'e de recherche INRIA Rh^one-Alpes, 655, avenue de l'Europe, 38330 MONTBONNOT ST MARTIN

Unit'e de recherche INRIA Rocquencourt, Domaine de Voluceau, Rocquencourt, BP 105, 78153 LE CHESNAY Cedex

Unit`e de recherche INRIA Sophia-Antipolis, 2004 route des Lucioles, BP 93, 06902 SOPHIA-ANTIPOLIS Cedex

Éditeur

INRIA, Domaine de Voluceau, Rocquencourt, BP 105, 78153 LE CHESNAY Cedex (France) http://www.inria.fr

ISSN 0249-6399 